\title{
Impact of nutrition on ageing the process. Bridging the Gap: the animal perspective $^{\succsim}$
}

\author{
Richard F. Butterwick* \\ WALTHAM Centre for Pet Nutrition, Waltham-on-the-Wolds, Melton Mowbray, Leicester LE14 4RT, UK \\ (Submitted 22 November 2013 - Final revision received 26 August 2014 - Accepted 19 September 2014)
}

\section{Abstract}

In pets, as in humans, there is increasing interest in interventions that promote 'health and well-being' into later life and extend these beyond their current limits. The purpose of this review was to assess the relevance of current knowledge of ageing in humans, described in a companion paper, as well as reviewing recent research on ageing in pet populations. The role of diet and other factors that influence the ageing process and ultimately lifespan in pets are highlighted in this review; in addition, future opportunities and challenges to further our understanding of the ageing process in pets are identified. Advancing knowledge of the fundamental biology of ageing will be key for the development and evaluation of strategies that extend both the quality and the quantity of lifespan in human and pet populations.

\section{Key words: Pets: Ageing: Dogs: Cats}

In human populations, lifespan has consistently increased over recent years, and this phenomenon shows no signs of abating, advancing on average 2 years per decade ${ }^{(1)}$. In principle, this is very good news; however, there are some down sides. Ageing in humans is accompanied by a progressive loss of physical capability and function, characterised as 'frailty', and this can lead to loss of mobility and independence, an increased need for support, creating challenges for public health policy, and increased demand for health and welfare systems.

The concept of ageing in pets is well established and recognised by owners and veterinarians. Terms such as senior and geriatric are widely used to describe the ageing pet; however, these life stages are not well defined and are usually assigned based on the chronological age of the pet. This approach is somewhat arbitrary and may not reflect the real 'biological' age of the pet or reflect some of the subtle changes that may be recognised by the owner such as activity, food intake, body weight, body composition, time spent sleeping or other changes in behaviour.

Research on ageing is highly relevant to the companion animal; after all, they are ageing too. Dogs and cats play a central role in many families; indeed, many are considered fully fledged 'family' members, and, as for any other family member, we aspire that they too have long and healthy lives.

\section{Changes in lifespan}

Reports on long-lived pets in the popular media and press indicate that some achieve remarkable longevity, with the oldest reported cat reaching an astonishing 38 years ${ }^{(2)}$. There is some evidence that lifespan is higher in pet or domesticated dogs and cats than in feral or 'wild' dog and cat populations. The average life expectancy in pet cats $(12-14 \text { years })^{(3,4)}$ is considerably higher than the reported median lifespan in feral cats (4.7) $)^{(5)}$. It is not known what factors contribute to the striking difference in lifespan between feral and pet cat populations, although accidents, diseases, parasites, availability of food and stresses of multiple and frequent pregnancies may contribute.

There is surprisingly little data on changes in the lifespan of pet populations over the past few decades. In part, this is due to a paucity of veterinary databases that record age of death and cause of death in representative pet populations. However, a recent report provides some data on lifespan changes in the North American pet population; over the past decade, average lifespan has increased by 1 and 0.5 years in cats and dogs, respectively ${ }^{(4)}$. The report did not provide any explanation for these changes; however, the provision

*Corresponding author: R. F. Butterwick, email richard.butterwick@effem.com

This article was published as part of the WALTHAM International Nutritional Sciences Symposium Proceedings 2013.

This paper was published as part of a supplement to British Journal of Nutrition, publication of which was supported by an unrestricted educational grant from Mars Incorporated. The papers included in this supplement were invited by the Guest Editor and have undergone the standard journal formal review process. They may be cited. 
of good health care and the availability of complete and balanced foods would have contributed to these gains.

\section{Biology of ageing}

In humans, it is thought that the accumulation of random molecular damage in the macromolecules of all cells is the major determinant of lifespan, and this results in agedependent defects in cellular function and reduced function at a tissue level which manifest as age-related frailty, disability and disease ${ }^{(1)}$. Many factors, both endogenous and exogenous, are considered to contribute to the cellular and tissue damage that contribute to ageing process. The development of interventions to prolong lifespan and/or halt the onset of age-related diseases is dependent on advancing knowledge and understanding of the fundamental biology of ageing.

\section{Factors influencing ageing in the companion animal}

Reproductive status has been shown to affect life span in pets. There is evidence that sterilisation is associated with increased lifespan in dogs and cats ${ }^{(4,6)}$. Sterilised dogs are less likely to die from infectious disease, trauma, vascular disease and degenerative disease and more likely to die from neoplasia and immune mediated disease. The nature of this association and potential mechanism is not understood; it could be mediated through a direct physiological pathway, or indirectly through behavioural changes, or a combination of both.

Body size in dogs is another factor known to influence lifespan. Dogs are unusual because the relationship between lifespan and body size is different from that of the interspecies association ${ }^{(7)}$, where generally larger animals live longer. In dogs, small breeds are expected to live much longer (from 10 to 14 years) than large and giant breeds (from 5 to 8 years) ${ }^{(8)}$.

A comparison of longevity of pet dogs and humans showed that at any chronological age, larger breed dogs are older in human year equivalents compared with their smaller counterparts ${ }^{(9)}$, dispelling the myth that 1 human year is equivalent to 7 dog years. A recent study ${ }^{(10)}$ investigated different models of ageing to see if they could identify attributes of the ageing process that could explain why large and giant breeds have a 'shorter' lifespan. They found no effect of breed size on baseline mortality, early life mortality or the age at which mortality started to increase (senescence). However, there was a clear positive relationship between the absolute rate of ageing and body size, indicating that large and giant breed dogs simply age faster than their smaller counterparts.

There is a growing body of evidence on the influence of diet on longevity and lifespan. Dietary energy restriction has been extensively studied in rodent models and has been shown to have a generally positive impact on lifespan. However, there is limited evidence that this effect translates to humans ${ }^{(1)}$. One study has investigated the effect of energy restriction in $\operatorname{dogs}^{(11)}$. A total of forty-eight Labrador retrievers from seven different litters were pair-matched (sex and weight) and assigned to one of two groups. One group (over fed) was initially fed ad libitum, although this was later restricted to avoid excessive weight gain, and the other group was restricted to $75 \%$ intake of their littermates (restricted). The study followed dogs over two decades and reported a significant increase in median lifespan (13.2 $\mathrm{v}$. 11 years) in the 'restricted' group. This was associated with a delay in the development of orthopaedic diseases, which is a common age-related issue in the breed studied. Body weight was also lower in the diet 'restricted' group; however, this difference did not appear to be due to differences in physical size, but as a result of increased adiposity in the 'over-fed' group, suggesting that being overweight may be a negative risk factor for lifespan. Energy restriction is unlikely to be a practical solution for most pets, or humans, and in reality, the consequences of excess energy intake, i.e. obesity, have far greater potential relevance to lifespan. In humans, the relationship between obesity and lifespan is well established, and in countries where obesity is now at 'epidemic' levels, such as the USA, there is the worrying phenomenon that the trend of increased lifespan over past decades will plateau and potentially reverse ${ }^{(12)}$. In dogs and cats, the impact of obesity is also known to be associated with various diseases $^{(13)}$, and there is also evidence that obesity shortens lifespan in $\operatorname{dogs}^{(14)}$.

\section{Conclusion}

Dogs and cats have had a long association with humans, and today they are the most popular companion animal. The nature of this association has changed from one where they have been a beast of burden, or even source of food, to a position where they are referred to as a 'companion animal', better reflecting the role they fulfil as 'family' members. As cherished family members, owners desire long and healthy lives for their pets. The development of interventions to extend health and well-being in pets and humans beyond their current limits will be dependent on gaining fundamental knowledge and understanding of the biological process that influence ageing. However, there are some specific challenges in companion animals that potentially limit progress in this field. Currently there is limited data on lifespan in pet populations, and this restricts our ability to track changes over time, or identify associations with any changes in lifestyle. In addition, 'cause of death' is often recorded as 'euthanasia' rather than the underlying medical condition. Addressing these data gaps may help identify specific issues that impact on lifespan in the companion animal.

\section{References}

1. Mathers JC (2013) Nutrition and ageing: knowledge, gaps and research priorities. Proc Nutr Soc 72, 246-250.

2. Guinness World Records (2010) Bantam, Reprint edition 2010, p. 320, Guinness World Records Limited.

3. Taylor EJ, Adams C \& Neville R (1995) Some nutritional aspects of ageing in dogs and cats. Proc Nutr Soc 54, 645-656.

4. Banfield Pet Hospital (2013) State of pet health 2013 report. http://www.stateofpethealth.com/Content/pdf/Banfield-Stateof-Pet-Health-Report_2013.pdf 
5. Levy J, Gale D \& Gale L (2003) Evaluation of a long-term trap-neuter-return and adoption program on a free-roaming cat population. J Am Vet Med Assoc 222, 42-46.

6. Hoffman JM, Creevy KE \& Promislow DEL (2013) Reproductive capability is associated with lifespan and cause of death in companion dogs. PLOS ONE 8, e61082.

7. Selman C, Nussey DH \& Monaghan P (2013) Ageing: it's a dog's life. Curr Biol 23, 451-453.

8. Michell AR (1999) Longevity of British breeds of dog and its relationships with-sex, size, cardiovascular variables and disease. Vet Rec 145, 626-629.

9. Patroneck GJ, Waters DJ \& Glickman LT (1997) Comparative longevity of pet dogs and humans: implications for gerontology research.J Gerontol A Biol Sci Med Sci 52, B171-B178.
10. Kraus C, Pavard S \& Promislow DEL (2013) The size-life span trade-off decomposed: why large dogs die young. Am Nat 181, 492-505.

11. Lawler FF, Larson BT, Ballam JM, et al. (2008) Diet restriction and ageing in the dog: major observations over two decades. Br J Nutr 99, 793-805.

12. Olshansky SJ, Passaro DJ, Hershow RC, et al. (2005) A potential decline in life expectancy in the United States in the 21st century. $N$ Engl J Med 352, 1138-1145.

13. German AJ (2006) The growing problem of obesity in dogs and cats. J Nutr 136, 1940S-1950S.

14. Salt C \& Morris P (2013) Associations between longevity and body condition in domestic dogs. In Proceedings of the WALTHAM International Nutritional Sciences Symposium, p. 52, Mars Incorporated. 